\title{
An Eddy Current Transducer Model for Image Restoration
}

Bennett R. Groshong

Griff L. Bilbro

Wesley E. Snyder

Center for Communications and Signal Processing Department Electrical and Computer Engineering North Carolina State University 


\begin{abstract}
We describe in this short paper a new imaging model for the spatial magnitude response of an absolute eddy current transducer to a flaw. This model is appropriate for image restoration purposes in that it captures the transducer response with sufficient accuracy for image restoration, yet is simple enough to be computationally practical. The model described in this paper is based on a simple resistive loop approximation to the transducer impedance changes induced by a flaw, and is efficiently implemented as layers of linear blurring functions and nonlinear point operations. The model is shown to accurately reflect the magnitude response of an absolute transducer to an EDM slot in a non-ferrous alloy. Furthermore, the model is shown to produce good restoration results for synthetic images of flaws. The model may be adapted to a particular combination of absolute transducer and surface flaw type by optimizing the model parameters, either by forming the partial derivatives with respect to the parameters and minimizing by gradient descent, or by a straightforward implementation of a neural net back-propagation algorithm. Other types of eddy current transducers may be modeled by simply modifying the spatial layers to combine the local terms appropriately for the geometry of the transducer.
\end{abstract}

KEYWORDS: Transducer model, layered nonlinear function, image restoration. 


\section{Introduction}

We describe in this short paper a new imaging model for the spatial magnitude response of an absolute eddy current transducer to a flaw. The transducer used in this work is an annular coil with a mean radius of approximately 34 mils wound on a ferrite core and enclosed in a ferrite shield bead. The transducer is operated at a frequency of two megahertz and is used to inspect metal surfaces for stress cracks with lengths in the 10 to 100 mil range. A nonlinearly distorted image of the the flaw is generated by sampling the response of the transducer on a four mil grid as it is scanned over the surface. We are interested in geometrically characterizing the flaw by restoring the distorted image.

Direct characterization of flaws from an eddy current image is complicated by the fact that the eddy current transducer has a nonlinear, large-radius point spread function (PSF) relative to the size of flaws of interest [6]. Linear image restoration methods such as the Wiener filter fail to model the nonlinear response of the sensor to flaws, which varies with the size of the flaw and its relative position [2]. The true response of an absolute eddy current transducer to a flawed surface is a complex phenomenon.

Modeling this response is an open research area, as indicated by the range of techniques discussed in recent journal articles. Iterative techniques such as Beissner's boundary element model [3] and the finite difference method of Auld et al [1] are useful for understanding the forward problem of simulating the sensor response, but their computational complexity makes them less than ideal for image restoration 
purposes. Integral methods $[7,10]$ are similar in that they seek to accurately model the electromagnetic fields and are also computationally complex, requiring solution over a large number of cells for a flawed surface.

What is needed for image restoration purposes is a model which captures the transducer response with sufficient accuracy for image restoration, yet is simple enough to be computationally practical. The model described in this paper accurately reflects the response of the sensor to a flaw, as indicated by the comparative images and plots in Section 4. It is based on a simple resistive loop approximation $[8,9]$ to the transducer impedance changes induced by a flaw, and is efficiently implemented as layers of linear blurring functions and nonlinear point operations. 


\section{Eddy Current Transducer Model}

The transducer forward model we have developed for flaw characterization is based on a simple model for the magnitude of the change in the impedance of the coil due to the presence of a flaw. The transducer coil induces a region of eddy current flow in the surface of the object to be inspected. This region mirrors the geometry of the coil, and may be approximated by an annular ring of current filaments flowing on the surface of the part $[4,8,9]$. The effect of each of these filaments on the transducer is modeled by considering the transducer coil as the primary and the filament as the secondary of a transformer $[8,9]$. A flaw in the surface changes the length of the circular path of the current filament, changing the resistance of the secondary and creating a voltage change in the primary [8, page 38$]$.

The response $g$ of the model at a point $(x, y)$ to a flaw $f$ is then a weighted sum of the path lengths of these current filaments, which may be written as

$$
g(x, y)=\int_{r=0}^{r_{\max }} w(r) \int_{\theta=0}^{2 \pi} \sqrt{r^{2}+\left(\frac{\partial f(x+r \cos \theta, y+r \sin \theta)}{\partial \theta}\right)^{2}} d \theta d r .
$$

The weight

$$
w_{r}=\frac{K}{\sqrt{2 \pi} \sigma_{p}} \exp \left\{-\frac{\left(r-r_{0}\right)^{2}}{2 \sigma_{p}^{2}}\right\}
$$

models the radial sensitivity profile of the transducer. This model is simple, analytic, and produces images that mimic those of the true transducer. However, it is not particularly amenable to iterative gradient descent image restoration, due to the large number of operations required for each point of response. One method of reducing this number is to approximate the derivative of $f$ in (1) by computing it for some 
fixed number of angles, then computing the local path length for each angle, and finally combining the intermediate results to get the complete response.

\section{Layered Nonlinear Representation}

Posing the transducer model as the composition of a number of layers of linear spatial operations and nonlinear point functions is a powerful technique, permitting efficient implementation of both the transducer model and its partial derivatives.

Nonlinear models written in layered form

$$
F=h^{L} * s^{L}\left(h^{L-1} * s^{L-1}\left(\cdots h^{0} * s^{0}(f) \cdots\right)\right)
$$

where the $h^{L}$ terms represent linear spatial operations (two dimensional convolutions), and are composed with the $s^{L}$ terms, which are nonlinear functions applied pointwise. Functions written in this way have a number of attractive features:

1. They may be implemented on a computer more efficiently than functions of the form of (1). The functions represented by the lower layers may be performed once, with their output combined appropriately by higher layers in a hierarchical manner, rather than repeating the complete set of calculations at each point.

2. The gradient of this form may be evaluated using the chain rule, and also written in layered form. Additional computational savings may be realized by re-using intermediate terms from the forward model when computing the gradient.

3. It has been shown [5] that the layered form is general in that a very wide range of functions may be represented. 
We write the transducer model (1) in this form by breaking the response into three layers.

The first layer computes the approximate change in the path the eddy current filaments take for a particular direction at a point. We model this directional response to a flaw for current filaments flowing at an arbitrary angle $\theta$ (See Figure 1) with respect to the $(x, y)$ axes by forming an anisotropically blurred Gaussian derivative function:

$$
\begin{aligned}
D(x, y, \theta) & =-\frac{d_{\theta}}{\sqrt{2 \pi} \sigma_{d}} \exp \left\{-\frac{\left(d_{\theta}\right)^{2}}{2 \sigma_{d}^{2}}\right\} \\
& \times \frac{1}{\sqrt{2 \pi} \sigma_{g}} \exp \left\{-\frac{\left(d_{\perp}\right)^{2}}{2 \sigma_{g}^{2}}\right\} .
\end{aligned}
$$

The term $d_{\theta}=x \cos \theta+y \sin \theta$ is the projection of the point $(x, y)$ onto a unit vector in the direction $\theta$, and $d_{\perp}=-x \sin \theta+y \cos \theta$ is the projection of the point $(x, y)$ onto a unit vector perpendicular to $\theta$. The first term models the local change in the current filament path at the point $(x, y)$, and is the first derivative of a Gaussian with spread $\sigma_{d}$ parallel to $\theta$. The second models the radial sensitivity profile of the transducer, and is a Gaussian with spread $\sigma_{g}$ perpendicular to $\theta$. This function is illustrated for a single $\theta$ in Figure 2.

The second layer transforms the directional derivative by the nonlinear point function

$$
s(\nu)=\sqrt{1+\nu^{2}}
$$

providing a local approximation to the filament path length per unit distance traveled in the plane in the direction $\theta$. 
The third layer linearly combines the local path length terms over an annulus, forming a weighted sum of the directional point path length terms at the appropriate angle and completing the point spread function of the model. The directional terms are combined using a set of polar Gaussian blurring functions

$$
\begin{aligned}
R(r, \phi) & =\frac{1}{2 \pi \sigma_{r} \sigma_{\phi}}\left\{\exp \left(-\frac{\left(r-r_{0}\right)^{2}}{2 \sigma_{r}^{2}}-\frac{\left(\phi-\phi_{0}\right)^{2}}{2 \sigma_{\phi}^{2}}\right)\right. \\
& \left.+\exp \left(-\frac{\left(r+r_{0}\right)^{2}}{2 \sigma_{r}^{2}}-\frac{\left(\phi-\phi_{0}-\frac{\pi}{2}\right)^{2}}{2 \sigma_{\phi}^{2}}\right)\right\}
\end{aligned}
$$

one for each term. One of these terms is illustrated in Figure 3. Note that the blurring function sums directional local path length terms from the regions on both sides of the annulus where the direction $\theta$ (See Figure 1) is approximately tangent.

The transducer model is implemented by calculating a set of convolution kernels for (4) and (6), one for each desired $\theta$ and $\phi$. The result is a transducer model written in the form of $(3)$ :

$$
g_{i j}=\sum_{n=0}^{N} k g_{n} * s\left(k d_{n} * f_{i j}\right) .
$$

where $k d_{n}$ is the kernel for the directional first derivative of a Gaussian (4) at angle $\theta_{n}=n \pi / N$, and $k g_{n}$ is the Gaussian blurring function (6) at angle $\phi_{n}=\theta_{n}+\pi / 2$. $N$ is chosen to be the minimum number of angles producing a smooth transducer response. $N=8$ was found to be sufficient. 


\section{Results}

The results given in this section illustrate the performance of the forward transducer model. Figure 4 is a magnitude image formed by combining the in-phase and quadrature components of the output of a General Electric Corporate Research and Development eddy current transducer in the usual way.

The imaging parameters were as follows:

- The transducer type was "sge9" This transducer is an absolute sensor with a single annular coil wound on a ferrite core and enclosed in a ferrite shield bead. The probe has a mean coil radius of 34 mils, and is operated at 2 megahertz.

- The imaged surface was a block of Rene-95 high nickel alloy, with a rectangular EDM slot 30 mils long by 3 mils wide by 15 mils deep.

- The image row and column sample spacing was 4 mils.

- The values displayed are the magnitude of the data samples.

- The image shown is 64 rows by 64 columns, with the image data videoscaled to 0 to 255 . The data is displayed using a linear gray scale map, with white representing 255 and black 0.

Figure 5 is a synthetic flaw image generated by the layered transducer model (7), using smooth derivative functions. The imaging parameters are:

- The original flaw image was 7 pixels long by 1 pixel wide by 15 units on a zero background in the center of the image. 
- The derivative kernels $k d_{n}$ were generated by numerically sampling the Gaussian derivative function (4) for all image points more than 1 percent of the peak magnitude. The deviations were $\sigma_{g}=1.7$ and $\sigma_{d}=1.0$ pixels.

- The radial kernel $k g_{n}$ was generated in the same manner as the derivative kernel, with $r_{0}=6.25, \phi_{0}=\theta+\pi / 2, \sigma_{r}=0.5$, and $\sigma_{\phi}=\pi r_{0} / \sqrt{2} N$, where $N=8$ is the number of angles.

- The image size and map are identical to Figure 4

A plot made by vertically slicing the two images in Figures 4 and 5 is shown in Figure 6 


\section{Discussion}

The absolute eddy current transducer model accurately reflects the response of a real transducer to a flaw, as shown in Figure 5. Furthermore, the layered model (7) is relatively efficient, requiring approximately $10^{3}$ multiplies per output image point, versus $10^{4}$ for the direct model (1). The number required for the layered model is not unreasonable, given that the point spread function of the transducer encompasses at least 700 pixels.

In addition to accurate forward modeling, the layered forward model has proven appropriate for image restoration. We have restored synthetic flaw images by forming the squared residual between the observed image and an estimate image distorted by the layered model, computing the gradient of the squared residual with respect to the estimate, and minimizing the residual by constrained gradient descent. An example restoration of the image shown in Figure 5 is shown in Figure 7. The smooth Gaussians and their derivatives used in the layered model have yielded stable restoration algorithms, giving us confidence that further work in this area will be fruitful.

The model may be adapted to a particular combination of absolute transducer and surface flaw by optimizing the model parameters $\sigma_{g}, \sigma_{d}, r_{0}, \sigma_{r}, \sigma_{\phi}$, and $N$ by forming the partial derivatives with respect to the parameters and minimizing by gradient descent. An equivalent minimization could be performed by a straightforward implementation of a neural net back-propagation algorithm.

Other types of eddy current transducers may be modeled by simply modifying the spatial functions $k g_{n}$ to combine the local terms appropriately for the geometry 
of the transducer. For instance, the spatial terms for a typical differential transducer would be similar to those in Figure 3, but the two lobes would have opposite sign and would vary in magnitude with the angle $\theta$. 


\section{References}

[1] B. A. Auld, S. R. Jefferies, and J. C. Moulder. Eddy-current signal analysis and inversion for semielliptical surface cracks. Journal of Nondestructive Evaluation, $7(1 / 2): 79-94,1988$.

[2] A. J. Bahr and B. A. Auld. An electromagnetic model for eddy-current imaging. Journal of Nondestructive Evaluation, 7(1/2):71-77, 1988.

[3] R. E. Beissner. Approximate model of eddy-current probe impedance for surfacebreaking flaws. Journal of Nondestructive Evaluation, 7(1/2):25-34, 1988.

[4] Hugo L. Libby. Introduction to Electromagnetic Nondestructive Test Methods. Wiley-Interscience, 1971.

[5] G. G. Lorentz. The 13-th problem of hilbert. In F. E. Bowler, editor, Proceedings of Symposia in Pure Mathematics: Mathematical Developments Arising from Hilbert Problems, pages 419-430, Providence, R. I., 1976. American Mathematical Society, American Mathematical Society.

[6] R. O. McCary, D. W. Oliver, K. H. Silverstein, and J. D. Young. Eddy current imaging. IEEE Transactions on Magnetics, MAG-20(5):1986-1988, September 1984.

[7] D. M. McKirdy. Recent improvements to the application of the volume integral method of eddy current modeling. Journal of Nondestructive Evaluation, 8(1):45-52, 1989.

[8] Robert C. McMaster, Paul McIntire, and Micheal L. Mester, editors. Nondestructive Testing Handbook, Volume 4. Electromagnetic Testing: Eddy Current, Flux Leakage, and Microwave Nondestructive Testing. American Society for Nondestructive Testing, 2nd edition, 1986.

[9] Atomic Energy of Canada Limited. Eddy Current Testing, volume 1. GP Courseware, 1987.

[10] Harold A. Sabbagh. A model of eddy current probes with ferrite cores. IEEE Transactions on Magnetics, 23(2):1888-1904, March 1987. 


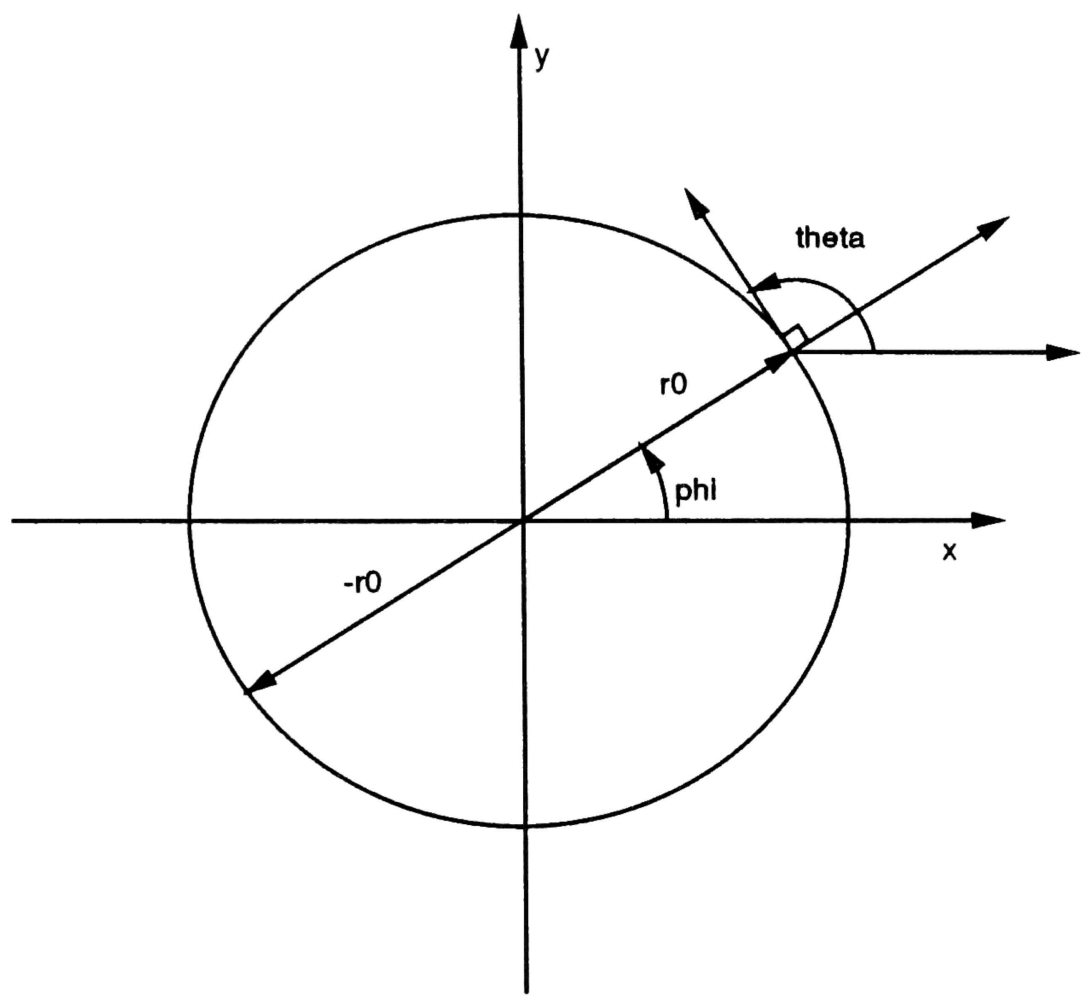

Figure 1: Illustration of $\theta$ and $\phi$ for transducer model.

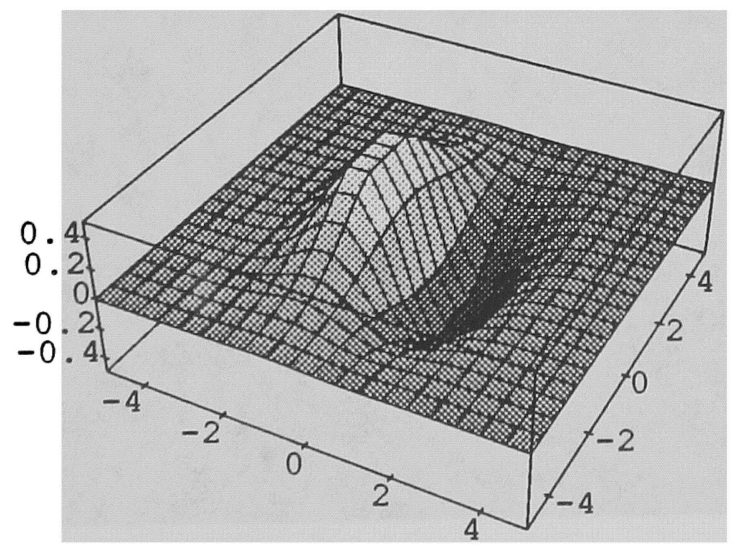

Figure 2: Blurred Gaussian derivative function.

Figure 1: Illustration of $\theta$ and $\phi$ for transducer model. 


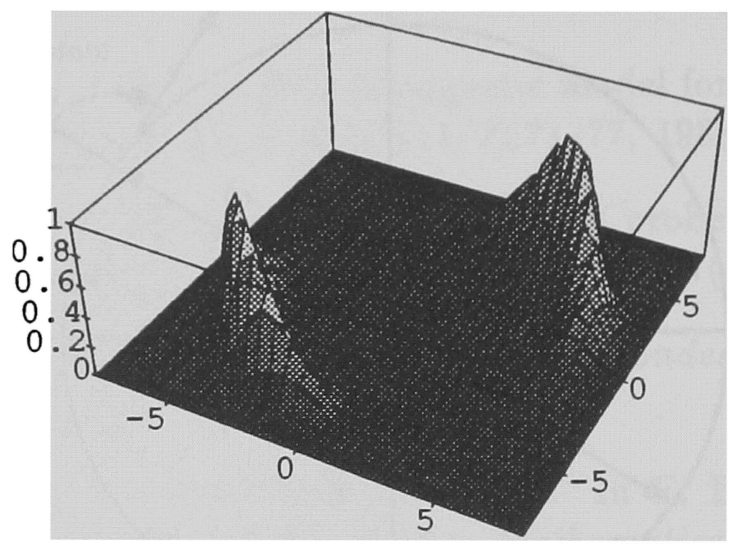

Figure 3: Gaussian blurring function.

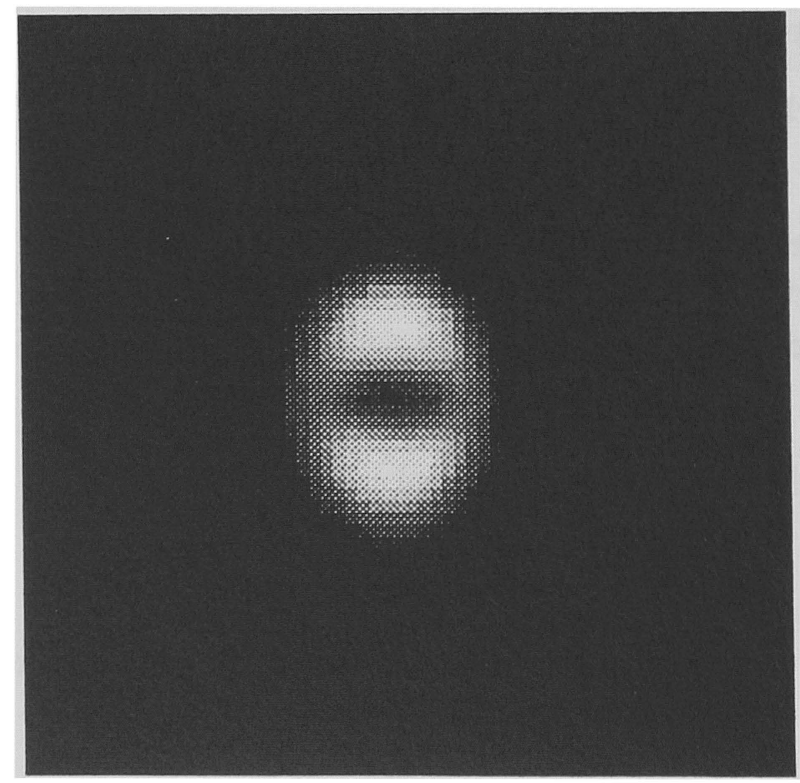

Figure 4: "edm15" magnitude image of EDM slot 30 mils long by 3 mils wide by 15 mils deep. 
Figure 5: Image of synthetic flaw using layered model with smooth derivative kernels.

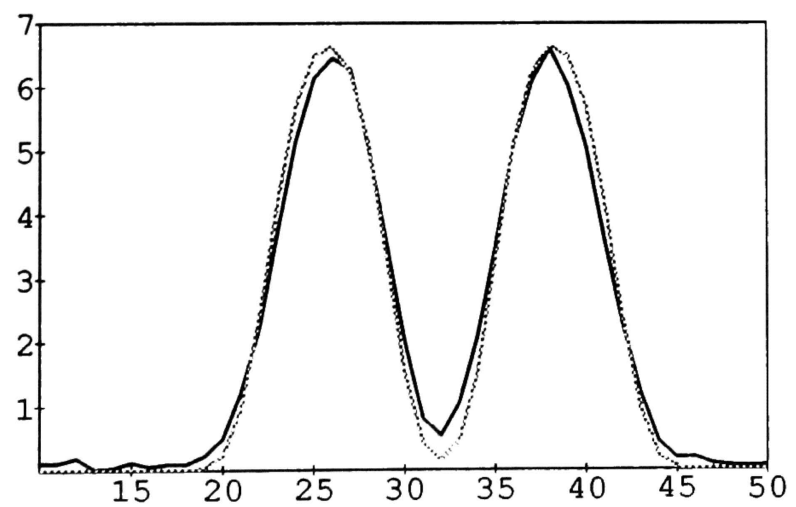

Figure 6: Vertical cross section of original absolute transducer image and forward model image of identical flaw. 


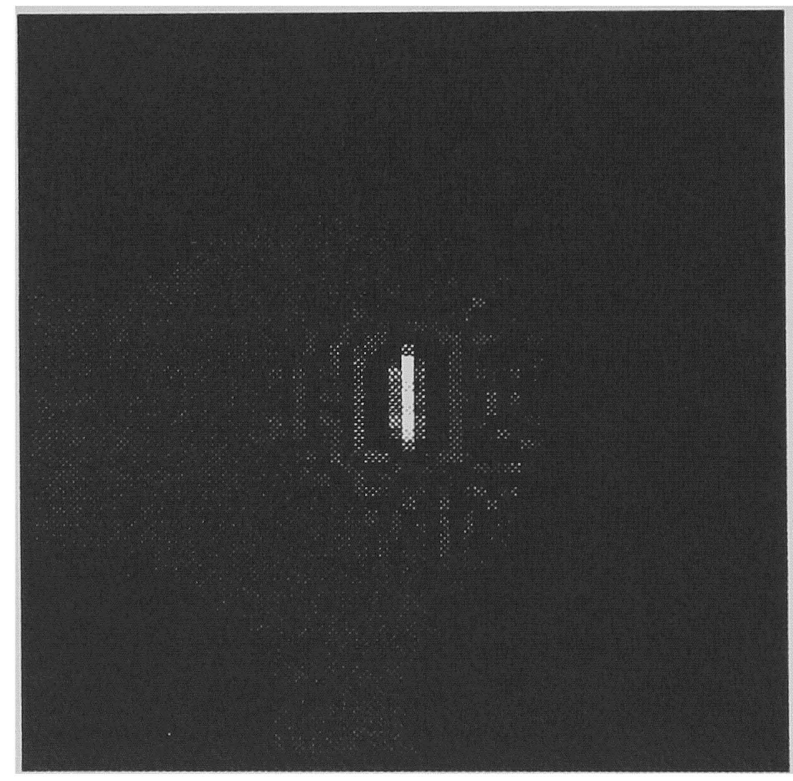

Figure 7: Image of restored synthetic flaw using layered model with smooth derivative kernels. 